\title{
Eficiencia de la aplicación de policloruro de aluminio en post-tratamiento de aguas industriales en palma de aceite
}

\section{Effectiveness of aluminum polychloride on oil palm industrial wastewater post-treatment.}

\author{
Yim J. Rodríguez-Díaz ${ }^{1 \oplus}$; Alcides A. Torregroza-Mozo ${ }^{1 \oplus}$; Franklin Mejía-Padilla ${ }^{1 *}$; \\ Jader J. Atencia-Vargas ${ }^{\circledR} ;$ Andrea C. Villero-González ${ }^{2 \oplus}$
}

Recibido para publicación: Junio 12 de 2016 - Aceptado para publicación: Mayo 24 de 2019

\section{RESUMEN}

Las plantas extractoras de aceite demandan grandes cantidades de agua en sus procesos, generando aguas residuales y residuos sólidos, ocasionando un impacto ambiental adverso. El objetivo de la investigación fue evaluar la eficiencia del Policloruro de Aluminio (PAC), en la remoción de parámetros contaminantes [Demanda química de oxigeno (DQO), grasas y aceites (G y A), turbidez, sólidos suspendidos totales (SST), sólidos suspendidos volátiles (SSV)] de las aguas residuales efluentes del tratamiento biológico de la empresa OLEOFLORES en Codazzi-Cesar. Se realizaron muestreos compuestos de las aguas residuales, conforme lo recomienda el Instituto de Hidrología, Meteorología y Estudios Ambientales; se realizó una caracterización y se determinó el rango óptimo de coagulante para la remoción de estos parámetros, encontrando valores iniciales en DQO, G y A, turbidez, SST y SSV de: 4111,20 mg.L- ${ }^{1}$, 737,26 mg.L- ${ }^{1}$, 939,33 NTU, 870 mg.L- ${ }^{1}$, 673,33 mg.L- ${ }^{1}$, respectivamente y una dosis óptima de: 3963 mg. L- ${ }^{1}$ de PAC, obteniendo porcentajes de remoción para DQO, SST y (G y A) de 75,79\%, 86,21\% y 98,8\%. Al comparar con Sulfato de Aluminio la remoción máxima obtenida para DQO, SST y G y A de: $34,14 \%, 11,88 \%$ y $85,64 \%$ respectivamente. Se concluye que el uso del PAC es una opción eficiente para el post-tratamiento de aguas residuales; además, contribuirá en el mejoramiento de la calidad de vida de las poblaciones que se beneficien del recurso hídrico afectado por los efluentes.

Palabras clave: Aguas industriales; Aceite de palma; Coagulante; Policloruro de aluminio; Post-tratamiento.

\footnotetext{
${ }^{1}$ Universidad del Cesar; Facultad de Ingenierías y tecnológicas.

${ }^{2}$ Universidad del Cesar; Facultad de Ingenierías y tecnológicas. Semillero de Investigación en Ingeniería ambiental y sanitaria (SIIAS).

*Autor para correspondencia: Franklin Mejía

Email: mejia5712@hotmail.com
}

ABSTRACT

Oil extraction plants demand large amount of water in their processes, generating wastewater, solid residuals and therefore negative environmental impact. The research objective was to evaluate the effectivity of aluminum polychloride (PAC) on contaminating parameter [Oxygen Chemical Demand (OCD), fats and oils (F and O), turbidity, total suspended solids (TSS) and volatile suspended solids (SSV)] removal from the wastewater effluent of OLEOFLORES' biological treatment in Codazzi - Cesar. Composed sampling of wastewater and characterization were performed according to IDEAM Initial values for OCD, $\mathrm{F}$ and $\mathrm{O}$, turbidity, TSS and VSS of 4111,20 mg.L-1 ${ }^{1}, 737,26$ mg.L-1 ${ }^{1}$ 939,33 NTU, 870 mg.L-1, 673,33 mg.L-1 respectively, were found. An optimal dose of 3963 mg.L- ${ }^{1}$ of PAC, removing 75,79\%, 86,21\% y 98,8\%. Compared with Aluminum sulphate were the maximum removal rate for OCD, TSS and $\mathrm{F}$ and $\mathrm{O}$ were $34,14 \%$, $11,88 \%$ y $85,64 \%$, respectively. It is concluded that the PAC is an effective option for wastewater treatment, contributing to improve life quality of life for populations who benefit from water affected by effluents.

Key words: Industrial water; Palm oil; Coagulant; Aluminium polychloride; Post-treatment.

\section{Cómo Citar}

Rodríguez-Díaz, Y., Torregoza-Mozo, A., Mejía-Padilla, F., Atencia, J. y Villero, A. 2019. Eficiencia de la aplicación de policroruro de aluminio en post-tratamiento de aguas industriales en palma de aceite. Temas Agrarios 24(2): 159-170 https://doi.org/10.21897/rta.v24i2.2068 


\section{INTRODUCCIÓN}

Para los países tropicales, la palma de aceite (Elaeis guineensis Jacq) representa una alternativa de excelente perspectiva para el futuro. Este cultivo produce 10 veces más del rendimiento de aceite proporcionado por la mayoría de los otros cultivos oleaginosos y con materiales genéticos más recientes la diferencia en rendimiento es cada vez mayor y los problemas de salud achacados a las grasas hidrogenadas tendrán que abrirle paso al aceite de palma para la fabricación de productos a base de origen vegetal (Sáens, 2006). El cultivo de palma de aceite en Colombia ha mantenido un aumento sostenido en las áreas de cultivo. A mediados de la década de los 60 existían 18.000 hectáreas en producción y hoy (año 2017) existen más de 377.662 hectáreas en 116 municipios del país distribuidos en cuatro zonas productivas, a saber: Zona Norte: departamentos de Magdalena, Norte del Cesar, Atlántico, Guajira, (95.879 hectáreas), Zona Central: departamentos, Santander, Norte de Santander, sur del Cesar, Bolívar, (119.107 hectáreas), Zona Oriental: departamentos de Meta, Cundinamarca, Casanare, Caquetá (147.700 hectáreas), y La Zona Occidental: en el departamento de Nariño (14.976 hectáreas), (FEDEPALMA, 2015). Para la producción de aceite de palma se llevan a cabo diferentes procesos que generan aguas residuales que son una fuente importante de contaminación (Nemerow y Dasgupta, 1998), ya que sus constituyentes alteran gravemente los ecosistemas.

El Ministerio de Ambiente y Desarrollo Sostenible presentó la Norma de Vertimientos Puntuales a Cuerpos de Aguas Superficiales y a los Sistemas de Alcantarillado Público, que busca reducir y controlar las sustancias contaminantes que llegan a los ríos, embalses, lagunas, cuerpos de agua naturales o artificiales de agua dulce, y al sistema de alcantarillado público, para de esta forma, aportar al mejoramiento de la calidad del agua y trabajar en la recuperación ambiental de las arterias fluviales del país (MINAMBIENTE, 2015), por lo que empezar a buscar alternativas para cumplir el marco legal de vertimientos es de suma importancia y urgencia.

Uno de los grandes productores de aceite de palma a nivel nacional es el grupo empresarial OLEOFLORES, el cual está ubicado al norte del municipio de Codazzi, en cercanía a la carretera que lo comunica con el municipio de San Diego. Tiene como objetivo crecer en la diversidad integrada de la cadena productiva del Aceite de Palma (Oleoflores, 2015), la cual genera aguas residuales provenientes de los procesos y operaciones unitarias; para el tratamiento de estos efluentes cuenta con un sistema de lagunas de oxidación Siendo eficaces para remover contaminantes orgánicos e inorgánicos, pero que en sus efluentes persisten contaminantes coloidales que deben ser tenidos en cuenta para el cumplimiento de la norma de vertimientos, por lo que es viable aplicar el proceso de coagulación-floculación para evaluar la eficiencia en la remoción de los mismos.

Los efluentes líquidos de una planta de procesamiento de aceite de palma consisten principalmente en lo siguiente: condensados de la esterilización, o el agua que resulta de la condensación del vapor utilizado para esterilizar los racimos, lodo de la clarificación, o los líquidos que quedan después de que los racimos han sido procesados y se han separado y extraído los aceites, entre otros (Oléagineux, 1982). Serios problemas ambientales se pueden originar si los desechos generados se continúan multiplicando y salen sin control o tratamiento alguno. Las plantas extractoras de aceite de palma están ubicadas principalmente cerca de ríos, debido a que se requieren grandes cantidades de agua para su operación. Es muy normal que durante los primeros días, las plantas extractoras descarguen el efluente directamente al río. Mientras más efluente 
contaminante se descargue en la cuenca del río, mayor es el impacto ambiental adverso ocasionado en el ecosistema del río. En la mayoría de los casos, el río eventualmente se vuelve séptico y seriamente se destruye la vida acuática y el hábitat (Azniidris y Aukay, 1995). Los efluentes generados en las plantas extractoras de aceite de palma representan un grave problema de contaminación por el alto contenido de sustancias orgánicas que presentan, esto debido a que las concentraciones de DQO son del orden de 60.000 mg.L- $^{1}$,

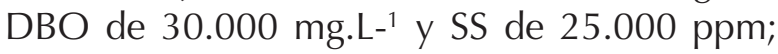
ricos en potasio (Díaz y vega, 2013). Esta alta carga orgánica requiere una elevada demanda biológica y química de oxígeno para su depuración, lo que altera el delicado equilibrio para la vida acuática en las corrientes, tanto superficiales como subterráneas. Tradicionalmente los tratamientos empleados para estos efluentes son la digestión anaeróbica (con o sin recirculación) seguida de un tratamiento aerobio mediante canales de oxidación, lodos activados o lagunas aireadas (Nemerow y Dasgupta, 1998).

Malacatus et al. (2017) estudiaron la generación de efluentes en el proceso de extracción de aceite crudo de Palma en el Ecuador. Postulando inicialmente que la generación de efluentes en el proceso de extracción de aceite de palma se relaciona con la capacidad de procesamiento de la planta, en este estudio, en Ecuador no se ha determinado un valor referencial de generación de efluente por tonelada de racimo de fruta fresca procesada (tRFF), el cual en Colombia se estimó en 0,84 $\mathrm{m}^{3} / \mathrm{tRFF}$. En ese estudio se analizaron datos de cinco plantas extractoras de aceite de palma ubicadas en la zona noroeste del país con el objetivo de establecer un valor promedio de generación de agua residual en relación a las toneladas de fruta fresca procesada. Como resultado se obtuvo que el valor promedio de generación de efluentes por tonelada de racimo de fruta fresca procesada en las cinco extractoras analizadas es $0,84 \mathrm{~m}^{3}$, el cual corrobora el promedio obtenido por el estudio de CENIPALMA Colombia, que es 0,84 $\mathrm{m}^{3} / \mathrm{tRFF}$. También, las etapas del proceso que generan mayor cantidad de efluente son: esterilización y extracción. Siendo estos los puntos críticos para el control y optimización del uso de agua. Otro resultado indica que se produjeron aproximadamente 2'371.471,2 $\mathrm{m}^{3}$ de efluentes de la industria de extracción de aceite crudo de palma en el año 2016, de acuerdo a las estadísticas de producción y el dato de generación de efluentes obtenido en el presente estudio.

Rodríguez et al. (2012) evaluó la eficiencia del coagulante orgánico quitosano como coagulante en el tratamiento de aguas asociadas a la producción de petróleo mediano(APPM).Serealizaronpruebasaescalade laboratorio de coagulación, floculación y sedimentación con tiempos de 1 minuto (mezcla rápida), 20 minutos (mezcla lenta) y 30 minutos, respectivamente. LasmuestrasdeAPPM, con valores de turbidez inicial de 8323 UNT, se recolectaron en el Patio de Tanque de Ulé, Costa Oriental del Lago de Maracaibo, estado Zulia. Se trabajó con quitosano comercial (QC) como coagulant evaluándose las concentraciones de 2, 3, 4, 5, 6 mg.L- ${ }^{1}$. Se determinaron los parámetros hidrocarburos, demanda química de oxígeno (DQO), turbidez, color, aceites y grasas (A y G), sólidos suspendidos totales (SST), sólidos suspendidos volátiles (SSV) y $\mathrm{pH}$ antes y después del tratamiento con el coagulante. El QC fue eficiente para la remoción de hidrocarburos, A y G, SST, SSV, turbidez y color presentes en las APPM, obteniéndose remociones superiores al 80\% para la concentración óptima de $4 \mathrm{mg} \cdot \mathrm{L}^{-}{ }^{1}$. El quitosano se presenta como alternativa para remover hidrocarburos de las APPM.

Por otra parte, Baquero et al. (2014) evaluaron la eficacia del quitosano como coagulante en el pos-tratamiento de aguas residuales municipales. Los resultados demostraron la eficacia del quitosano para remover valores del parámetro DQO, en el post-tratamiento de las aguas residuales 
municipales del STAR EL SALGUERO de Valledupar, la cual presenta, según el informe final de las caracterizaciones de los sistemas de aguas residuales el Tarullal y el Salguero de la ciudad de Valledupar al igual que sus fuentes receptoras, río Guatapuri y río Cesar, realizado por la empresa de servicios públicos de Valledupar - EMDUPAR S.A. E.S.P. en septiembre del 2013 valores promedio en el afluente de 428,95 $\mathrm{mgO}_{2} \mathrm{~L}^{-1}$, los datos arrojados por la caracterización realizada en la investigación en el efluente del STAR fue de 203,904 mgO2 L-1 , lo cual nos indica que el sistema de tratamiento de aguas residuales "STAR EL SALGUERO" remueve con el sistema de lagunas el 52,46\%. Al aplicar el tratamiento de coagulación-floculación con el coagulante natural (quitosano), con una dosis de 80 mg.L- ${ }^{1}$ (esta fue la dosis de coagulante que obtuvo el mayor valor de remoción de DQO) se obtiene una remoción del 57,63\% del valor de DQO del efluente disminuyendo notablemente la carga vertida al rio Cesar.

De Armas y Ramírez (2015) evaluaron el desempeño y eficiencia de dos coagulantes químicos: sulfato de aluminio y policloruro de aluminio (PAC), y dos naturales: moringa (Moringa oleífera) y cardón guajiro (Lemaireoreus griseus) en la remoción de nutrientes y sedimentos del efluente de la planta de tratamiento de aguas residuales conformado por lagunas de estabilización El Salguero ubicada en el municipio de Valledupar, Cesar, Colombia. Los ensayos se llevaron a cabo con muestras de agua procedentes del río Cesar recogidas en el efluente del sistema de tratamiento. La turbidez varía desde 25 NTU hasta 67 NTU durante la época de desarrollo de la investigación.

Se obtuvo que el sulfato de aluminio pese a no alcanzar la turbidez exigida por la normatividad colombiana (Decreto 3930/2010, Articulo $\mathrm{N}^{\circ} 72$ Turbidez $\geq 5$ NTU) es un coagulante que remueve más del $80 \%$ de los parámetros de DQO, DBO y algas. El policloruro de aluminio es adecuado para disminuir significativamente la turbidez y los SST en un 92\%, además presentó la mejor viabilidad económica de los tratamientos; y para la disminución de fosforo total resultaron efectivos los tratamientos con coagulantes naturales moringa y cardón, los cuales presentaron una remoción de 90,35\%. Se concluye que los coagulantes evaluados presentan un potencial de remoción de los parámetros analizados, los resultados avalan a la Moringa oleífera como una posible alternativa natural en la potabilización de las aguas, ya que garantiza no sólo lograr con éxito el proceso de coagulación, sino también, la ausencia de residuos.

Finalmente, Diaz et al. (2017), realizaron un estudio de tratamiento físico-químico mediante adición de coagulante y floculantes, con el objetivo de determinar un programa químico para las aguas residuales oleosas provenientes del proceso de separación por gravedad en una refinería de petróleo. Los ensayos del proceso se efectuaron en equipo de Pruebas de Jarras (Jar Test), donde se evaluaron los productos comerciales policloruro de aluminio (PAC), sulfato de aluminio y Sintec D50 con cinco floculantes diferentes.

El programa químico seleccionado se evaluó con los fluidos a tres temperaturas para conocer su sensibilidad a este parámetro y la energía de mezcla en la coagulación y la floculación. Se determinó el programa químico y las características operacionales para el tratamiento físico-químico con PAC, obteniendo una remoción de más de 93\% para la materia suspendida y de $96 \%$ para hidrocarburos totales para el par coagulante/ floculante seleccionado.

Por lo anterior, el propósito de esta investigación fue determinar la eficiencia de un Post-tratamiento Físico-Químico aplicando un coagulante inorgánico, Policloruro de Aluminio (PAC), en la remoción de turbidez, sólidos suspendidos totales (SST), sólidos suspendidos volátiles (SSV), Grasas 
y Aceites (G y A) y Demanda Química de Oxígeno (DQO) contenidas en las aguas residuales efluentes del tratamiento biológico. Para el alcanzar este objetivo se caracterizaron las aguas residuales efluentes, se simulo en el laboratorio el proceso de Coagulación-Floculación para determinar los rangos óptimos, luego se determinó la dosis óptima y la eficiencia del tratamiento en los parámetros DQO, SST y G Y A, para el cual el PAC resultó eficiente como post-tratamiento para todos los parámetros evaluados, obteniendo porcentajes de remoción para DQO, SST y (G y A) de 75,79\%, $86,21 \%$ y $98,80 \%$ respectivamente.

\section{MATERIALES Y MÉTODOS}

La investigación tuvo lugar en el laboratorio de Ingeniería Ambiental y Sanitaria de la Universidad Popular del Cesar, Valledupar Colombia, sede Sabanas. Las aguas para producción de aceite de palma (APAP) se recolectaron manualmente en el efluente de planta de tratamiento biológico, ubicada en la empresa OLEOFLORES S.A. del municipio de Agustín Codazzi, Cesar. Se realizaron muestreos compuestos conforme al instructivo para la toma de muestras de aguas residuales del Instituto de Hidrología, Meteorología y Estudios Ambientales "IDEAM" (IDEAM, 2007), dicha muestra se compuso en 8 horas según el plan de muestreo; los cuales fueron tomados a la salida de la última laguna del tratamiento biológico de las aguas residuales provenientes de los procesos de producción de dicha empresa. Las alícuotas se almacenaron en recipientes plásticos de $20 \mathrm{~L}$ y se refrigeraron a $4^{\circ} \mathrm{C}$ para su conservación. (NTC-ISO-5667-10 1995).

La evaluación de la coagulación se llevó a cabo utilizando un aparato de Prueba de Jarra modelo PB-700; se agregó 1 L de APAP, a cada uno de los seis vasos de precipitado de $1000 \mathrm{~mL}$, tomando uno de estos como control. Posteriormente, se procedió a agregar el coagulante, al iniciar el mezclado rápido
(125 rpm, 2min); se agregaron en cinco de los vasos de precipitado las diferentes dosis de coagulante usando una pipeta, se aplicó luego el mezclado lento (40 rpm, $20 \mathrm{~min}$ ), se finalizó el proceso con la fase de sedimentación (40 min). Los ensayos se realizaron por triplicado para PAC y sulfato de aluminio, a una temperatura de $25 \pm 1{ }^{\circ} \mathrm{C}$. Los parámetros fisicoquímicos de cada una de las muestras, se determinaron antes y después del tratamiento. Para determinar la concentración óptima se consideró la menor concentración del coagulante que remueva el mayor valor de DQO. Las muestras para la determinación de los parámetros de estudios después del tratamiento, fueron tomadas del sobrenadante de cada jarra en dos frascos de $250 \mathrm{~mL}$, discriminados así, para la determinación de $\mathrm{G}$ y A se tomó una muestra por cada jarra y fue conservado con ácido sulfúrico analítico (Merk 97\% de pureza), mientras que para determinar los parámetros turbidez, pH, DQO, SST, SSV se tomó otro recipiente de la misma capacidad, ambos fueron conservados a $4^{\circ} \mathrm{C}$.

Para la caracterización y efectividad del proceso de tratamiento de las APAP se determinóla turbidez, pH, DQO, SST, A y G y SSV fueron establecidos por los métodos estándar (APHA, 1992). Se analizaron antes y después del tratamiento de coagulación para evaluar la efectividad del Policloruro, siguiendo la misma metodología antes mencionada. Todos se describen a continuación.

Para la determinación de la DQO se empleó el método titulométrico. Se prepararon tubos de digestión agregando 1,5 mL de solución de dicromato de potasio J.T Baker 0,025N, 2,5 $\mathrm{mL}$ de ácido sulfúrico (H2SO4) (Merk 97\% de pureza). Luego se adicionaron en cada tubo $2,5 \mathrm{~mL}$ de la muestra, para el control y la muestra de las APAP también se preparó un blanco agregando 2,5 mL de agua destilada. Los tubos se colocaron en un equipo para digestión (EACTOR-DQO 115-230 V/50-60HZ); por reflujo a $150^{\circ} \mathrm{C}$ durante $2 \mathrm{~h}$. Luego se realizaron las titulaciones con FAS. 


$$
\text { DQO como mg O } 2 / \mathrm{L}=\frac{(A-B) \times N \times 8 \times 1000}{\mathrm{~mL} \text { de muestra }}
$$

Donde:

$\mathrm{A}=$ Promedio de $\mathrm{mL}$ de FAS utilizado para los blancos digeridos

$B=m L$ de FAS utilizado para la muestra

$\mathrm{N}=$ Normalidad del FAS

8 = Peso equivalente del Oxígeno.

Para la determinación de los SST y SSV se empleó el método gravimétrico, usando una balanza analítica HR 250A-DE252 SN\# 6A7602606 SERIE: 255190 (Toledo Al2O4), papel de filtro con fibra de vidrio y cápsulas de aluminio.

Se filtraron por succión $25 \mathrm{~mL}$ de muestra a través papel de filtro previamente pesado en una cápsula de aluminio hasta peso constante $\left(\mathrm{P}_{1}\right)$, las cápsulas se introdujeron en un horno (Memmert) con un rango de temperatura de $103-105^{\circ} \mathrm{C}$ durante una hora. Luego se dejaron enfriar en un desecador hasta peso constante $\left(\mathrm{P}_{2}\right)$. Seguidamente las cápsulas se introdujeron en una mufla (Thermolyne SYBRON 48000 Furnace) a $550^{\circ} \mathrm{C}$ durante 5 minutos y luego se dejaron enfriar en un desecador hasta peso constante (P3). Las siguientes ecuaciones permitieron determinar las concentraciones de SST y SSV respectivamente, en las muestras de agua residual.

$$
\begin{aligned}
& \mathrm{SST}(\mathrm{mg} \mathrm{L}-1)=\left(\mathrm{P}_{2}-\mathrm{P}_{1}\right) \times 106 / \mathrm{VM} \\
& \mathrm{SSV}(\mathrm{mg} \mathrm{L}-1)=\left(\mathrm{P}_{2}-\mathrm{P}_{3}\right) \times 106 / \mathrm{VM}
\end{aligned}
$$

Dónde:

P1: Peso de la cápsula, a temperatura ambiente, (g).

P2: Peso del residuo + cápsula, a $103-105^{\circ}$ C, (g).

P3: Peso del residuo + cápsula, a $500^{\circ} \mathrm{C}$, (g). VM: Volumen de Muestra $(\mathrm{mL})$.

Para la determinación de los A y G se utilizó el método gravimétrico. Se agregaron $170 \mathrm{~mL}$ de muestra en un balón de separación, luego se adicionaron $2 \mathrm{~mL}$ de ácido clorhídrico (Merck $\quad 37 \%$ de pureza) y $20 \mathrm{~mL}$ de Xileno (Applichem Pareac) como solvente. Posteriormente se realizó el proceso de extracción, se repitió la extracción dos veces más con $5 \mathrm{~mL}$ del solvente, recolectando en un vaso de precipitado previamente secado y pesado $(\mathrm{P} 4)$, previa filtración en papel de filtro conteniendo $\mathrm{Na}_{2} \mathrm{SO}_{4}$, la fase orgánica. Se dejó evaporar hasta alcanzar peso constante (P5). La siguiente ecuación permitió determinar la concentración de A y G.

$$
\text { A y } G(m g L-1)=\left(P_{5}-P_{4}\right) \times 106 / V M
$$

Dónde:

P4: Peso del vaso de precipitado (g).

P5: Peso del vaso de precipitado + muestra (g).

VM: Volumen de Muestra, $(\mathrm{mL})$.

El pH se determinó a través del método potenciométrico, usando un $\mathrm{pH}$-metro (Orion Research 611), con electrodo de vidrio, calibrado con soluciones buffers de $\mathrm{pH} 4$ y 7. Se midieron $200 \mathrm{~mL}$ de muestra en un matraz de $250 \mathrm{~mL}$, se introdujo electrodo del $\mathrm{pH}$-metro hasta obtener la lectura constante.

En la medición de turbidez se tomaron 25 $\mathrm{mL}$ de la muestra previa agitación y se llevaron al turbidimétro Hatch, a una longitud de onda de $450 \mathrm{~nm}$, calibrado con el blanco (agua destilada), arrojando la medida de la turbidez. La preparación de la solución coagulante se utilizó el Policloruro de Aluminio (Grupo Transmerquim $100 \%$ de pureza) comercial al $50 \%$ V/V, el cual se diluyó hasta conseguir una concentración del 1\% V/V. Se trabajó con el rango de concentraciones que según las pruebas preliminares, por medio de ensayo y error, arrojaron los mejores resultados. Adicionalmente, para comparar la eficiencia del PAC, se trabajó con Sulfato de aluminio (PQP 98\% de pureza) en solución al 1\% V/V.

Se utilizó un diseño completamente al azar con una significancia de $\alpha=0,05$ de confianza para el análisis de varianza y probar la igualdad de medias entre los tratamientos. Al existir diferencias entre los tratamientos se realizó una prueba de medias por el método de Dunnett (Gutiérrez, 2008). 


\section{RESULTADOS Y DISCUSIÓN}

Se debe tener en cuenta la revisión de los tratamientos biológicos aplicados por la empresa y su eficiencia ya que las aguas provenientes de la última laguna de oxidación del tratamiento biológico que aplica el grupo empresarial OLEOFLORES presentan las características mostradas en la tabla 1 (Los valores presentados son los promedios respectivos), con la desviación estándar encontrada entre un periodo de muestreo y otro; para el vertimiento final de estas aguas residuales no se recomienda descargarlas en un cuerpo de agua superficial (estos efluentes se pueden reusar en agua de riego y ser reciclado en procesos de compostaje) debido a la entrada en vigencia de la nueva resolución 0631 del 2015 sobre vertimientos, exceptuando el pH y la DBO5, además de los parámetros SSV y Turbidez, que no se encuentran normados. Si a los efectos formulados por Azniidris y Aukay S. 1995 y los de Nemerow y Dasgupta, 1998, se suman los volúmenes de agua que se generan en la industria, los cuales se formulan en la investigación de Malacatus et al. (2017), para las plantas extractoras de aceite de palma, es importante que la empresa OLEOFLORES S. A. empiece buscar alternativas para el debido cumplimiento de la resolución 0631 del 2015 en lo que respecta a los futuros vertimientos del agua residual a las fuentes hídricas, entre los cuales la presente investigación plantea una posible solución al problema.

Rodríguez y col (2012) al caracterizar sus aguas obtuvieron parámetros importantes como una DQO de 3553 mg.L- ${ }^{1}$ que es incluso más bajo que la mostrada en la tabla, sin embargo en los A y G obtuvieron 2050 mg.L- ${ }^{1}$, el contenido presentado es mayor que el que se obtuvo en la presente investigación el mismo comportamiento se registra en la presencia de SST 1117 mg.L-1 ${ }^{1}$, Siendo esta investigación la más oportuna para comparaciones futuras.

Se determinó la relación DBO5/DQO para justificar el tratamiento fisicoquímico a realizar en el agua residual. Obteniendo el siguiente resultado:

$$
\frac{D B O 5}{D Q O}=\frac{459,00}{4111,20}=0,112
$$

Tabla 1. Caracterización fisicoquímica del efluente de la última laguna del tratamiento biológico realizado por el grupo empresarial OLEOFLORES al agua residual, comparado con la normatividad actual de vertimientos

\begin{tabular}{|c|c|c|c|}
\hline Parámetro & Unidad & $\begin{array}{c}\text { Cantidad en el efluente de la } \\
\text { última laguna }\end{array}$ & $\begin{array}{c}\text { Valor límite máximo permisi- } \\
\text { ble para vertimientos a cuer- } \\
\text { pos de aguas superficiales }{ }^{3}\end{array}$ \\
\hline $\begin{array}{l}\text { Demanda química de } \\
\text { oxigeno (DQO) }\end{array}$ & mg.L-1 de O2 & $4111,20 \pm 1285,03$ & 1500,00 \\
\hline $\begin{array}{c}\text { Demanda bioquímica de } \\
\text { oxigeno (DBO5) }\end{array}$ & mg.L- ${ }^{1}$ de O2 & 459 & 600,00 \\
\hline $\begin{array}{c}\text { Grasas y aceites } \\
(\mathrm{G} \text { y } \mathrm{A})\end{array}$ & mg.L-1 & $737,25 \pm 173,94$ & 20,00 \\
\hline Potencial de hidrogeno $(\mathrm{pH})$ & Unidades de $\mathrm{pH}$ & $7,62 \pm 0,16$ & 6,00 a 9,00 \\
\hline $\begin{array}{l}\text { Sólidos suspendidos totales } \\
\text { (SST) }\end{array}$ & mg.L-1 & $870,00 \pm 60$ & 400,00 \\
\hline $\begin{array}{l}\text { Sólidos suspendidos volátiles } \\
\text { (SSV) }\end{array}$ & mg.L-1 & $673,33 \pm 50$ & No registra \\
\hline Turbidez & NTU & $939,33 \pm 68,32$ & No registra \\
\hline
\end{tabular}

${ }^{3}$ Resolución 0631 de 2015 
Según Crites y Tchobanoglous (2000), con un resultado de 0,112 se considera que el agua residual es de difícil biodegradación, por lo que es justificado un tratamiento fisicoquímico para la remoción de los parámetros contaminantes. Es importante resaltar que la investigaciónsetrataunaguaquehapasadoporun tratamientobiológicoanterior, el cual necesitaser complementado, para lo cual, una posibilidad sería un tratamiento fisco-químico.

Ya anteriormente Rodríguez et al. (2012) ha investigado el comportamiento de la coagulación con un coagulante natural en aguas con cargas contaminantes aceitosas, por otro lado, Baquero et al. (2014) aplicó procesos afines como pos-tratamiento de aguas municipales. Finalmente, Díaz y col (2017) y De armas y Ramírez (2015) de forma individual han investigado el comportamiento del PAC y el sulfato de aluminio en aguas asociadas a la producción de petróleos y aguas municipales respectivamente.
Inicialmente se establecieron diferentes rangos para determinar el rango óptimo, estos rangos se fueron acotando de acuerdo al comportamiento resultado de la experimentación, fueron corroborados y finalmente se presentan los que presentaron el mejor comportamiento para la totalidad de los parámetros evaluados. Para el PAC resultó estar en 3444, 3690, 3936, 4182, 4428, y 4674 mg.L- ${ }^{1}$, para el sulfato de aluminio el rango encontrado es de 2000, 2200, 2400, 2600, 2800, y 3000 mg.L- ${ }^{1}$ de Sulfato de aluminio, siendo la dosis óptima de 3963 para el PAC y de 2400 mg.L-1 ${ }^{1}$ para el Sulfato de Aluminio.

Según la tabla 2, la DQO promedio del

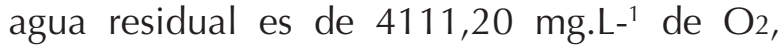
está por encima del valor establecido en la resolución 0631 del 2015. Después del tratamiento con PAC, se reduce cumpliendo con el valor límite máximo permisible en 5 de las 6 dosis probadas, presentándose la mejor remoción en la dosis de 4182 mg.L- ${ }^{1}$ de PAC.

Tabla 2. Resultados de los tratamientos realizados en el agua residual industrial de la empresa OLEOFLORES para determinar la dosis óptima de PAC, comparados con la normatividad actual de vertimientos.

\begin{tabular}{|c|c|c|c|c|c|c|}
\hline $\begin{array}{c}\text { DOSIS } \\
\left(\mathrm{mg}^{-L^{-}}\right) \text {de PAC }\end{array}$ & $\mathrm{pH}$ & $\begin{array}{c}\text { DQO } \\
\left(\mathrm{mgO}_{2} \mathrm{~L}^{-1}\right)\end{array}$ & $\begin{array}{c}\text { SST } \\
\left(\mathbf{m g} . \mathrm{L}^{1}\right)\end{array}$ & $\begin{array}{c}\text { SSV } \\
\left(\mathbf{m g} . \mathrm{L}^{-1}\right)\end{array}$ & $\begin{array}{c}\text { G y A } \\
\text { (mg.L-1) }\end{array}$ & $\begin{array}{l}\text { TURBIDEZ } \\
\text { (NTU) }\end{array}$ \\
\hline 3444 & 7,32 & 1563,16 & 200 & 120 & 32,35 & 72,92 \\
\hline 3690 & 6,93 & 1382,91 & 160 & 120 & 11,76 & 57,22 \\
\hline 3936 & 6,63 & 1191,09 & 126,6 & 86,6 & 8,82 & 36,89 \\
\hline 4182 & 6,71 & 995,41 & 146,6 & 100 & 52,94 & 30,81 \\
\hline 4428 & 6,65 & 1004,77 & 120 & 80 & 70,59 & 42,77 \\
\hline 4674 & 6,62 & 1058,97 & 120 & 90 & 13,73 & 46,34 \\
\hline $\begin{array}{l}\text { LIMITE MAXIMO } \\
\text { NORMADO }\end{array}$ & 6,00 a 9,00 & 1500,00 & 400,00 & No registra & 20,00 & No registra \\
\hline $\begin{array}{l}\text { MUESTRA } \\
\text { INICIAL }\end{array}$ & 7,62 & 4111,20 & 870 & 673,3 & 737,2 & 939,33 \\
\hline
\end{tabular}


La muestra tenía una concentración de 870,00 mg.L-1 de SST inicialmente, la cual está muy por encima del valor establecido en la normatividad. Después del tratamiento con PAC, la concentración de SST se reduce cumpliendo con el valor límite máximo permisible en todas las dosis probadas, presentándose la mejor remoción en las dosis de 4428 mg.L- ${ }^{1}$ y 4674 mg.L- ${ }^{1}$ de PAC. La concentración de G y A de 737,25 mg.L-1 ${ }^{1}$ está por encima del valor establecido en la normatividad.

Después del tratamiento con el rango mencionado de PAC, la concentración de G y A se reduce considerablemente, logrando cumplir con el valor límite máximo permisible en tres de las dosis probadas, presentándose la mejor remoción en la dosis de 3936 mg.L-1 de PAC, dicha dosis es el punto de quiebre.

Con respecto al $\mathrm{pH}$, el agua residual muestreada tiene un $\mathrm{pH}$ de 7,62 que cumple con los valores establecidos en la normatividad, y aún después del tratamiento con PAC, los valores presentan una disminución con relación a la inicial, manteniéndose dentro de los valores límites máximos permisibles en todas las dosis probadas.

Además de los parámetros normados se encontraron reducciones significativas en turbidez y solidos suspendidos volátiles (SSV) pasando de una turbidez inicial de 939,33 NTU hasta una mínima de 30,81 NTU presentándose la mejor remoción en la dosis de 4182 mg.L- ${ }^{1}$ de PAC; y una reducción de SSV de 665,00 mg.L- ${ }^{1}$ hasta 80,00 mg.L- ${ }^{1}$ presentándose la mejor remoción en la dosis de 4428 mg.L-1 ${ }^{1}$ PAC.

Después del tratamiento con el rango encontrado de Sulfato de Aluminio, la DQO de la muestra se reduce de 4111,20 mg.L-1 hasta 2708 mg.L- ${ }^{1}$ de O2, pero no logra cumplir con el valor límite máximo permisible en ninguna de las 6 dosis probadas.
Los SST se reducen hasta una concentración de 767 mg.L-1 ${ }^{1}$, lo cual es poco significativo y no logra cumplir con el valor límite máximo permisible en ninguna de las 6 dosis probadas.

La concentración de G y A se reduce considerablemente, pasando de 737,25 mg.L- ${ }^{1}$ hasta 106 mg.L- ${ }^{1}$, sin embargo, no se logra cumplir con el valor límite máximo permisibleen ninguna de las dosis probadas. Después del tratamiento con Sulfato de Aluminio, los valores de $\mathrm{pH}$ presentan un aumento con relación a la inicial, pero se mantienen dentro de los valores límites máximos permisibles en todas las dosis probadas. Con respecto a los parámetros no normados el Sulfato de Aluminio con una dosis óptima de 2600 mg.L- ${ }^{1}$ remueve hasta un 65 y $52 \%$ de turbidez y SSV respectivamente.

La eficiencia del PAC en la remoción de DQO es mayor que la eficiencia de remoción de del Sulfato de Aluminio, aunque el Sulfato de Aluminio presenta su mejor comportamiento con dosis menores, los porcentajes de remoción de $\mathrm{DQO}$ en el rango de dosis aplicadasconPACvaríanentre61,98\% y 75,79\%; porcentajes que están por encima de los presentados por el Sulfato que varían entre 13,18 \% y $34,14 \%$. Rodríguez et al. (2012) alcanzaron remociones de hasta un $57 \%$ de DQO con un coagulante natural, lo cual superan al Sulfato, pero presentan una menor remoción que el PAC, en lo que respecta a la comparación con aguas aceitosas, se aclara que dicha comparación se realiza para aguas de naturaleza aceitosa, aunque difieren en sus orígenes. La investigación de De armas y Ramírez (2015) plantea un comportamiento distinto para aguas municipales, para este parámetro el sulfato de aluminio resulta mejor con una remoción de $84,71 \%$ y el PAC con $81,91 \%$, dicha remoción es muy similar en este caso.

La eficiencia del PAC en la remoción de SST es mayor que la eficiencia de remoción del Sulfato de Aluminio. Los porcentajes de 
remoción de SST en el rango de dosis aplicadas con PAC varían entre $77,01 \%$ y $86,21 \%$; y los del Sulfato varían entre varían entre $3,45 \%$ y $11,88 \%$. Rodríguez et al. (2012) en su investigación, para los SST se removieron en un rango de $77 \%$ a $89 \%$., en los cuales se presenta un comportamiento muy parecido del quitosano y el PAC, ambos superiores al porcentaje de remoción presentado por el sulfato de aluminio. En ese sentido, la eficacia del PAC en la remoción de $\mathrm{G}$ y $\mathrm{A}$ es mayor que la eficiencia de remoción del Sulfato de Aluminio. Los porcentajes de remoción de G y A en el rango de dosis aplicadas con PAC varían entre 90,43\% y 98,80\%; y los del Sulfato varían entre $74,47 \%$ y $85,64 \%$. Rodríguez et al. (2012) después de la sedimentación obtuvieron un remanentede A y G menor a 400 mg.L-1, equivalente a una remoción del $80 \%$ por el coagulante natural quitosano, dicho porcentaje se equipara a la remoción del sulfato aluminio en la presente investigación, pero sigue estando por debajo de la remoción presentada por el PAC.

Para la turbidez el PAC presenta mayor remoción que el Sulfato de Aluminio. Los porcentajes de remoción de turbidez en el rango de dosis aplicadas con PAC varían entre $92,24 \%$ y $96,72 \%$; y los del Sulfato varían entre varían entre $47,62 \%$ y $65,05 \%$. Rodríguez et al. (2012) trabajaron con una turbidez de 8323 NTU removiendo hasta un $95 \%$ con un coagulante orgánico, lo que muestra eficiencia de los coagulantes en remoción de turbidez para guas aceitosas. Por su parte De armas y Ramírez (2015) corroboran una vez más que el PAC presenta mejor rendimiento en la remoción de turbidez de aguas municipales como coagulante primario sobre el sulfato de aluminio.

Resultados parecidos se obtuvo en la remoción de SSV donde también es mayor que la eficiencia de remoción del PAC con respecto al Sulfato de Aluminio. Los porcentajes de remoción de SSV en el rango de dosis aplicadas con PAC varían entre 81,95 \% y $87,97 \%$; y los del Sulfato varían entre varían entre $6,77 \%$ y 51,88 \%. Rodríguez et al. (2012) en su investigación, para los SSV se removieron en un rango de $77 \%$ a $89 \%$., en los cuales se presenta un comportamiento muy parecido del quitosano y el PAC, y ambos superiores al porcentaje de remoción presentado por el sulfato de aluminio.

Como consideración final, al comparar el PAC con el sulfato y el coagulante orgánico quitosano presentado en los antecedentes, en todas las investigaciones comparadas $y$ sumando la de Díaz y col (2017), los coagulantes son una buena alternativa para la remoción de los principales parámetros mostrados. Además el PAC resulta la mejor alternativa en la investigación de Díaz y col (2017), la segunda mejor en la investigación de De armas y Ramírez (2015) y la mejor en la presente investigación, igualando y superando a todos los coagulantes orgánicos presentados en los antecedentes mencionados.

Aunque tradicionalmente los tratamientos empleados para estos efluentes son la digestión anaeróbica (con o sin recirculación) seguida de un tratamiento aerobio mediante canales de oxidación, lodos activados o lagunas aireadas (Nemerow y Dasgupta, 1998); los resultados apoyados por los antecedentes presentan resultados favorables para un post-tratamiento con la coagulación, en este caso la mejor alternativa presentada resulta ser el PAC.

\section{CONCLUSIONES}

Las características actuales del agu a residual efluente del tratamiento biológico de la empresa OEOFLORES pueden ser ajustadas a la normatividad vigente con el post-tratamiento con PAC, si se pretende realizar vertimientos a cuerpos de agua superficial; la caracterización de estos efluentes depende del momento en que son tomadas las muestras ya que existe variabilidad significativa entre un 
muestreo y otro; los parámetros que presentan una desviación más significativa son G y A, SST y DQO. La dosis óptima de PAC para remoción de parámetros fisicoquímicos (DQO, SST, G y A, pH, turbidez, SSV) es 3936 mg.L- ${ }^{1}$, a pesar que las dosis óptimas difieren entre algunos parámetros, se tuvo en cuenta la dosis que legalmente cumpliera con los límites máximos permisibles de todos los parámetros establecidos en la normatividad Colombiana, resultando ser además la mejor dosis ambiental y económicamente. La remoción en los distintos parámetros evaluados, hacen del PAC una excelente opción para el post-tratamiento de las aguas efluentes del tratamiento biológico en la empresa del grupo empresarial OLEOFLORES.

El PAC es eficiente para la remoción de DQO, SST y G y A con un porcentaje de remoción de hasta $75,79 \%$, 86,21\% y $98,80 \%$ respectivamente, además el $\mathrm{pH}$ permanece dentro de los límites en la dosis óptima; estos porcentajes son suficientes para que los parámetros cumplan con los valores límites máximos permisibles establecidos en la resolución 0631 del 2015 de vertimientos a cuerpos de agua superficial. El PAC es más eficiente que el sulfato de aluminio para la remoción de todos los parámetros evaluados.

\section{Conflicto de intereses}

Los autores declaran que es un trabajo orginal y no existío conflicto de intereses de ningún tipo en la elaboración y publicación del manuscrito.

\section{REFERENCIAS}

APHA. 1992. Métodos normalizados para el análisis de aguas potables y residuales. Díaz de Santos, Madrid.

Azniidris, y Aukay, S. 1995. Manejo de efluentes de las plantas extractoras de aceite de palma - proceso de la más avanzada tecnología. Recuperado de:

http://publicaciones.fedepalma.org/index. php/palmas/article/viewFile/509/509
Baquero, M., Ortiz, J. y Rodríguez Y. 2014. Eficacia del quitosano como coagulante en el pos-tratamiento de aguas residuales municipales. Memorias del II Seminario de Ciencias Ambientales Sue-Caribe \& VII Seminario Internacional de Gestión Ambiental. Colombia. ISBN: 978-9589244-64-7

Crites, Tchobanoglous. 2000. Tratamiento de aguas residuales en pequeñas poblaciones. McGRAW-HILL, Bogotá.

De Armas, D. y Ramirez, L. 2015 Remoción de nutrientes mediante coagulantes naturales y químicos en planta de tratamiento de aguas residuales, Valledupar Colombia. Revista de Investigación Agraria y Ambiental. Colombia. Vol 6, No 2.

Diaz, M., Rivas, L., Fernandez, D., Salazar, D., Miller, S. y Maza, N. 2017. Selección de programa químico de tratamiento para aguas residuales oleosas. Revista centro azúcar. Vol 44, No 2.

Diaz, R. y Vega J. 2013. Efecto de la variación de la carga orgánica en el desempeño de un reactor uasb (upflow anaerobic sludge blanket) tratando efluentes de una planta extractora de aceite de palma. Revista ambiental agua aire y suelo. Colombia. Vol 4, No 1.

Fedepalma. 2015. Evolución histórica anual del fruto procesado, el aceite de palma y el palmiste.

Gutiérrez, H. 2008. Análisis y diseño de experimentos. México DF, McGRAWHILL/INTERAMERICANA EDITORES, S.A. p62-73

IDEAM. 2007. Instructivo para la toma de muestras de aguas residuales. Grupo laboratorio de calidad ambiental. Colombia. 
Malacatus, P. Guerrero, B. y Llerena, G. 2017. Generación de efluentes en el proceso de extracción de aceite crudo de Palma en el Ecuador. Revista dominio de las ciencias. Ecuador. Vol 3, No 4.

Ministerio de ambiente y desarrollo sostenible. Resolución 0631 del 2015. Por la cual se establecen los parámetros y los valores límites máximos permisibles en los vertimientos puntuales a los cuerpos de agua superficiales y a los sistemas de alcantarillado público y se dictan otras disposiciones. Bogotá D.C.

Nemerow, N. y Dasgupta, A. 1998. Tratamiento de vertidos industriales y peligrosos. Ediciones Díaz de Santos, Madrid. P481482
Norma técnica colombiana NTC-ISO-5667-10. 1995. ICONTEC. Bogotá.

Oléagineux. 1982. Internet vol. 37 No. 7.

http://temporal-fedepalma-ojs.biteca. com/index.php/palmas/article/ viewFile/41/41

Oleoflores. 2015. Recuperado de: http://www. oleoflores.com

Rodriguez, Y., Oñate, H., Gitierrez, E. y Caldera, Y. 2012. Eficiencia del quitosano como coagulanteen el tratamiento de aguas asociadas a la producción de petróleo mediano. Revista arbitrada venezolana. Venezuela. Vol 7, No 2.

Sáens, L. 2006. Cultivo de la palma africana guía técnica. Internet. 